\title{
EDITORIAL
}

\section{COVID-19 related Pharmacy education and workforce challenges in Zimbabwe}

\author{
Margaret Siyawamwaya $^{1}$, Donald Chuma ${ }^{2}$ (D) Tsitsi Monera-Penduka ${ }^{3}$ (D) \\ ${ }^{1}$ University of Zimbabwe Department of Pharmacy and Pharmaceutical Sciences, Faculty of Medicine and Health Sciences, Zimbabwe \\ ${ }^{2}$ Midlands State University, Department of Clinical Pharmacology, Faculty of Medicine, Zimbabwe \\ ${ }^{3}$ University of Zimbabwe Department of Primary Health Sciences, Faculty of Medicine and Health Sciences, Zimbabwe
}

\section{Correspondence}

Tsitsi Monera-Penduka

University of Zimbabwe

Department of Primary Health Sciences

Faculty of Medicine and Health Sciences

Zimbabwe

moneratg@yahoo.co.uk
While the national lockdown has presented many challenges for both pharmacy education and pharmacy practice in Zimbabwe, pharmacy educators and policymakers have risen to the occasion, and their response has advanced the profession. This editorial describes the impact of the COVID-19 lockdown on pharmacy education and the pharmacy workforce and highlights some of the pragmatic responses and an outlook for the future of pharmacy in Zimbabwe.
Zimbabwe has two schools of pharmacy, the University of Zimbabwe (UZ) and the Harare Institute of Technology (HIT), both located in the capital city of Harare. The two schools have a total annual intake of approximately 150 students. Pharmacy practice is regulated by the Pharmacists Council of Zimbabwe (PCZ) which maintains the national register of all practicing pharmacists and pre-registration trainee pharmacists (Health-Professions Act, 2004). In addition, all dispensing pharmacists require a practicing license issued by the national drug regulatory authority, the Medicines Control Authority of Zimbabwe, as stipulated in the Medicine and Allied Substances Control Act 15:03 (law.co.zw, 2020). Both registrations are renewable annually, and the demonstration of good standing and continuing professional development is required.

\section{Pharmacy education}

The government's lockdown directive of 30th March 2020 led to the temporary campus closure of all higher and tertiary institutions, including the two schools of pharmacy (Mukeredzi, 2020). As a result, in-person teaching had to be terminated in the middle of the academic year which typically runs from August to July. The administrators of both institutions responded in a timely manner by mandating the use of Moodle-based electronic learning management systems that were already in place to ensure the continuation of learning (University of Zimbabwe, 2020). At the UZ, faculty were required to attend at least one face to face or virtual refresher training of an upgraded version of the e-learning system. At the HIT, arrangements were made to provide lecturers with mobile telephone lines preloaded with data to facilitate off-campus access to the e-learning system.

Refresher training was not conducted at the HIT, and this was noted by lecturers as one of the challenges to the effective use of the e-learning system. Restricted access to university campuses also meant that learners could not use university facilities for learning, which proved to be a major challenge to the successful implementation of this initiative. Most families were financially constrained due 
to COVID-19 related loss of income and were unable to support data costs and acquire personal smart devices for off-campus access to the e-learning system. In addition, limited Internet coverage and bandwidth were mentioned as difficulties, particularly by students living away from the major towns and cities. Alternative conferencing options such as Zoom and Skype which require a simultaneous Internet connection were also not feasible for the same reasons, and in addition, because of the challenge of load shedding in most residential areas.

Due to these challenges, lecturers had to reimagine the mode of delivery for the instructional material and assessment without compromising the quality of education offered. To accommodate learners facing challenges with accessing the e-learning system, some lecturers resorted to distributing course material on memory sticks or via personal e-mails, Telegram or WhatsApp groups, which can be supported by simpler electronic gadgets and consume less Internet bandwidth. Lecturers were committed to redoing or adapting most of the instructional materials which were initially designed for interactive in-person use, by incorporating voice notes with explanations. Group discussions are an effective way of learning and understanding the vast amount of work associated with the pharmacy undergraduate degree. The WhatsApp platform has been the most effective platform for learning. It proved particularly useful for organising learners into small groups, which were otherwise hindered by lockdown period restrictions, and it supported peer discussions to help learners grasp important concepts and ensure continued supervision of undergraduate research projects (Matyanga, Dzingirai, \& Monera-Penduka, 2020).

Pharmacy education constitutes a large proportion of experiential learning activities across different stages of the curriculum, such as attachment at a public health institution, community and hospital pharmacy attachments, as well as clinical inpatient and outpatient rotations. While the online learning approaches provided a solution to the delivery of theoretical course material, University administration did not provide alternatives for laboratory experiments, clinical ward rotations, and practice-based attachments. In particular, reagents and equipment that had been ordered for experiments were affected by delays in the movement of cargo even before the national lockdown. To address this, the School of Pharmacy at the UZ will fully integrate a virtual dispensing practice skill laboratory into the Pharmacy Practice courses in the next academic year. The software, freely available from the Monash University, was collaboratively adapted for the Zimbabwean pharmacy practice setting but has only been partially integrated into the curriculum (Monera-Penduka et al., 2018). The same software will also be collaboratively adapted to teach HIV and infectious disease (HIV/ID) pharmacotherapy clinical practice with support from the International Pharmaceutical Federation (FIP). This will build capacity for clinical academic educators in Zimbabwe and enable the continuation of experiential learning activities and development of clinical skills during the COVID-19 pandemic and any future pandemics without unnecessary exposure to those involved in learning and teaching.

In a series of virtual surveys conducted by the UZ's School of Pharmacy, it was apparent that self-study at home was taxing for many learners given the challenges described above. Some disclosed that they were demotivated and depressed by the lack of clarity as to when, and how, exams would be conducted. This information was useful in the development of guidelines and Standard Operating Procedures (SOPs) for addressing mental health challenges for both students and staff. The UZ plans to roll out periodic virtual mental health sessions for students to address some of these challenges.

\section{Pharmacy workforce}

Retail and hospital pharmacy was considered an essential service and pharmacies had the option to remain open over the lockdown period. The profession contributed to the national COVID-19 response by managing the supply chain product quality of both COVID-19 and nonCOVID-19 related pharmaceutical products, including protective equipment, disinfectants, and hand sanitiser. In addition, they responded to numerous requests for drug information, virtually and in-person, and from both clients and other healthcare professionals. This has been key in keeping patients safe and avoiding misinformation from the barrage of information from a wide range of sources. The extended role of the pharmacist that has been highlighted in the pandemic will go a long way in advocating for pharmacy workforce policy.

There were also several pharmacy workforce developmentrelated challenges. Similar to pharmacy education, both pre-registration training and continuous professional development activities, which are traditionally conducted face to face, have been derailed by the restrictions in movement. The Council has two sets of qualifying exams in place, one after the pharmacy education and the second at the end of the two-year early career training period. This will serve the Council well for quality assurance of the compromised pharmacy education and training in the pandemic. In order to maintain the 
standard of practice, the PCZ resolved to maintain the points required for re-registration; however, they have relaxed the requirement to have the points be acquired from specific sources, and be from different categories of CPD (PCZ memo, 2020). It is highly likely that many practitioners will undertake COVID-19 related CPD activities in their area of practice in response to current needs (Dzingirai et al., 2020).

\section{Future outlook}

The COVID-19 lockdown experience has inarguably led pharmacy educators and policymakers in Zimbabwe to re-imagine and revise existing policies to ensure continuity of learning. The resulting changes and pragmatic solutions have, in the process, advanced pharmacy education and workforce development in the country. In particular, the facilitators and barriers to e-learning in the local context are well known and can be tackled head-on.

The FIP Workforce Development Goal (WDG) number three emphasises the need to ensure the quality of the workforce through continuous development and adequate education and training. Strategies will need to be implemented to ensure that, in future, the quality of the pharmacy workforce will not be affected by public health crises. This can be achieved by embracing technological advancements in training and education. Schools of Pharmacy will need to complement the University's efforts to deliver course content online by integrating dry and virtual laboratories in their practical courses. This is particularly important as the supply chain for pharmaceutical reagents is likely to remain stressed for the foreseeable future. Contrary to the belief that academia is a late-career choice, capacity building efforts should be implemented to encourage young pharmacists to pursue careers in academia. This will diversify the pharmacy workforce in academic settings in line with FIP WDG number one, and strengthen the capacity to implement e-learning methodologies.

Acceptance of a broad range of COVID-19 related CPD activities has demonstrated prioritisation of needs-based activities and helped to advance CPD pharmacy workforce development goals in line with FIP WDG number nine. The PCZ will also need to review the requirements for re-registration to include points acquired from a broader range of categories and sources, including a broader range of virtual activities.

\section{References}

Dzingirai, B., Matyanga, C.M.J., Mudzviti, T., Siyawamwaya, M., \& Tagwireyi, D. (2020). Risks to the community pharmacists and pharmacy personnel during COVID-19 pandemic: perspectives from a low-income country. Journal of Pharmaceutical Policy and Practice, 13(1), 42. https://doi.org/10.1186/s40545-020-00250-2

Health Professions Act. (2004). Chapter 27:19 Health Professions Act, Acts 6/2000, 22/2001,14/2002, 28/2004. Available at: http://www.mdpcz.co.zw/wp-content/uploads/2016/09/Health-P rofessions-Act.pdf

law.co.zw. (2020). Medicine and Allied Substances Control Act, Chapter 15:03 . Available at: https://www.law.co.zw/download/ medicines-and-allied-substances-control-act/

Matyanga, C.M.J., Dzingirai, B., \& Monera-Penduka, T.G. (2020). Virtual supervision of pharmacy undergraduate research projects during the COVID-19 lockdown in Zimbabwe. Pharmacy Education, 20(2), 13-14. https://doi.org/10.46542/pe.2020.202.1314

Monera-Penduka, T.G., Sukwe, T., Peters, P., Beaumont, K., Sewell, K., \& Marriott, J. (2018). Collaborative development of a virtual Pharmacy Practice skills laboratory at the University of Zimbabwe School of Pharmacy. Pharmacy Education, 18(1), 268-274

Mukeredzi, T. (2020). Under pressure, government closes universities and schools. Available at: https://www.university worldnews.com/post.php?story $=20200323065236567$

PCZ. (2020). PCZ Memo - Internal communication. PCZ, June 2020

University of Zimbabwe. (2020). Memo - Internal communication. University of Zimbabwe, May 2020 